\title{
Transcription factor IIE binds preferentially to RNA polymerase IIa and recruits TFIIH: a model for promoter clearance
}

\author{
Mary E. Maxon, James A. Goodrich, and Robert Tjian \\ Howard Hughes Medical Institute, Department of Molecular and Cell Biology, University of California, \\ Berkeley, California 94720 USA
}

\begin{abstract}
The basal factor TFIIE is an important component of the RNA polymerase II transcription machinery. In our efforts to determine the role of TFIIE in the transcription process, we have begun to define the interactions between TFIE and other basal transcription factors. Here we report that TFIIE binds selectively to the nonphosphorylated form of RNA polymerase II (IIa) and that this interaction is mediated by the 56-kD subunit of TFIE. Additional binding studies reveal that TFIIE can interact with TBP as well as TFIID and that this interaction is mediated primarily via the $56-\mathrm{kD}$ subunit. Our studies indicate that TFIIE also interacts with both subunits of TFIIF and with TFIIH, a multisubunit basal factor reported to catalyze RNA polymerase II CTD phosphorylation. Protein affinity assays demonstrate that TFIIE binds directly to ERCC-3, a DNA repair protein associated with TFIIH. More importantly, TFIIE affinity resin can selectively isolate transcriptionally competent TFIIH from a partially purified preparation and thereby may recruit TFIIH to the transcription complex in vivo. These multiple interactions between TFIIE, RNA Pol II and TFIIH support a model in which TFIIE plays a role in promoter clearance as well as in the recruitment of proteins required for transcription-coupled DNA repair.
\end{abstract}

[Key Words: RNA polymerase II; TFIIE; TFIIH; transcription factor; promoter clearance; DNA repair]

Received December 9, 1993; revised version accepted January 14, 1994.

Transcription of protein-encoding genes in eukaryotes is directed by RNA polymerase II (RNA Pol II) and a complement of auxiliary factors. One class of factors is comprised of sequence-specific DNA-binding proteins that recognize unique promoter elements and subsequently regulate transcription (for review, see Mitchell and Tjian 1989|. A second class of factors, the basal transcription factors, exerts its effects through the core elements of RNA Pol II promoters (TATA box and initiator motif), and is required for accurate initiation and transcription at most, if not all, promoters (for review, see Zawel and Reinberg 1993). Although the basal factors (TFIIA, TFIIB, TFIID, TFIIE, TFIIF, and TFIIH), have been identified based on their ability to reconstitute transcription in vitro, the mechanism by which many of the individual factors contributes to the formation of an active transcription complex remains unclear. For example, it is unlikely that all of these ancillary basal factors are required for the initiation of transcription. Instead, it is likely that some of them direct initiation while others act during the promoter clearance or elongation steps of the reaction. Additionally, any and all of these defined steps of transcription may be targets for specific regulatory factors that could serve to accelerate or inhibit the various steps of transcription directly. The recent availability of purified recombinant basal factors provided us with an opportunity to carry out a more rigorous analysis of the activities and interactions of the basal factors with other members of the transcription complex, thereby providing a clearer understanding of the events that govern mRNA synthesis.

Previous work had shown that human TFIIE is a heterotetramer comprised of two subunits of 34 and $56 \mathrm{kD}$ (TFIIE-34 and TFIIE-56; Inostroza et al. 1991). The subunits of TFIIE are in a 1:1 ratio in the heterotetramer and appear to be required in the same ratio for optimal reconstituted transcription and entry into a partially formed transcription complex as measured by gel mobility-shift analysis (Peterson et al. 1991). Although TFIIE has been considered one of the basal or general factors required to initiate transcription at all RNA Pol II promoters, several studies suggest that at least some promoters can be transcribed without TFIIE (Parvin et al. 1992; Tyree et al. 1993). In addition, recent abortive initiation assays that are able to discriminate between factors required for initiation versus promoter clearance and elongation suggest that TFIIE may not be required for the initiation step at some promoters (J. Goodrich, in 
prep.). Similarly, studies of elongation complexes suggest that TFIIE is released from the transcription complex once the elongation phase of transcription is reached (D. Reinberg, pers. comm.). The role of TFIIE is therefore of considerable interest because it does not appear to be required for either initiation or elongation, and yet it is clear from the available data that it is an essential transcription factor for most templates that have been tested.

In this report, we have attempted to determine the role of TFIIE during transcription. In particular, we have assayed the ability of TFIIE subunits to make protein-protein contacts with all of the known components of the basal transcriptional machinery. Interestingly, the two subunits of TFIIE interact selectively with multiple targets and our findings suggest a model in which TFIIE plays a role in TFIIH recruitment, transcription complex stabilization, promoter clearance, and association of ancillary enzymatic complexes involved in DNA repair.

\section{Results \\ TFIIE interacts with the nonphosphorylated form of RNA Pol II}

Assembly of the preinitiation complex, as shown by gel mobility shift analysis (Buratowski et al. 1989; Flores et al. 1991), is a highly ordered process that is thought to begin with the recognition of promoter elements by TFIID, which consists of the TATA-binding protein (TBP) and at least eight associated TAFs (TBP-associated factors)(Dynlacht et al. 1991; Tanese et al. 1991; Zhou et al. 1992). The binding of TFIIB and TFIIA in turn stabilizes the TFIID/DNA complex (Buratowski et al. 1989; Maldonado et al. 1990). It is this structure that is recognized by the TFIF/RNA Pol II complex (Flores et al. 1991). TFIIE is then thought to enter the transcription assembly, and subsequently, TFIIH and TFIIJ associate to form a transcription competent complex (Inostroza et al. 1991; Flores et al. 1992). Because the entry of TFIIE into the preinitiation complex is preceded by several other factors, there exist many potential interaction surfaces that may participate in the union of TFIIE with the preinitiation superstructure. The components of the complex that enter immediately prior to TFIIE are TFIIF and RNA Pol II, and these are thought to enter together. In light of previous studies that showed a cosedimentation of TFIIE and RNA Pol II (Reinberg and Roeder 1987a; Flores et al. 1989), we first asked whether TFIIE could recognize and bind RNA Pol II to form a stable complex in solution. Our experimental strategy was designed to also identify which subunit(s) of TFIIE contacted RNA Pol II and which form of RNA Pol II would be associated with TFIIE.

RNA Pol II can be purified from HeLa cells in both its phosphorylated (IIo) and nonphosphorylated (IIa) forms (Reinberg and Roeder 1987a; Lu et al. 1991). The IIo form of the enzyme possesses a multitude of phosphorylated residues at the carboxyl terminus of its largest subunit, which consists of 52 heptapeptide repeats (Corden et al. 1985). Extensive phosphorylation of this carboxy-terminal domain (CTD) results in a decreased mobility of the largest subunit $(240 \mathrm{kD})$ with respect to the nonphosphorylated large subunit $(200 \mathrm{kD})$. The RNA Pol II purified from HeLa cells used in our experiments is a mixture of the IIo and IIa forms of the enzyme as shown in lanes 1 and 2 of Figure 1 . To detect direct binding between TFIIE and RNA Pol II, we have carried out coimmunoprecipitation experiments with recombinant TFIIE subunits. The large subunit of TFIIE and the nonphosphorylated IIa form of RNA Pol II are efficiently coimmunoprecipitated with antibodies against TFIIE-56 either in the presence or absence of TFIIE-34 (Fig. 1, lanes 4 and 5, respectively), with more RNA Pol II being coimmunoprecipitated in the presence of TFIIE-34. In striking contrast, we found very little of the phosphorylated IIo form of RNA Pol II coprecipitated with TFIIE-56 even though the starting material contains a molar excess of the phosphorylated form of the enzyme. In a control experiment to demonstrate that the Ilo form had not been inadvertently converted to the IIa form by contaminating phosphatases in our protein preparations, we assayed the supernatants of the immunoaffinity reactions and found that the phosphorylated species was present and intact (Fig.1, lanes 6-8) and that the nonphosphorylated species had been significantly depleted (Fig. 1, lane 7). These results indicate that the $56-\mathrm{kD}$ subunit of TFIIE can interact directly and selectively with the IIa form of RNA Pol II. This finding is consistent with previous studies demonstrating that it is primarily the nonphosphorylated form of RNA Pol II that is recruited to the transcription complex at the promoter (Laybourn and Dahmus 1990; Lu et al. 1991). In similar experiments using exclusively RNA Pol IIo as the input, a very small amount of the phosphory-

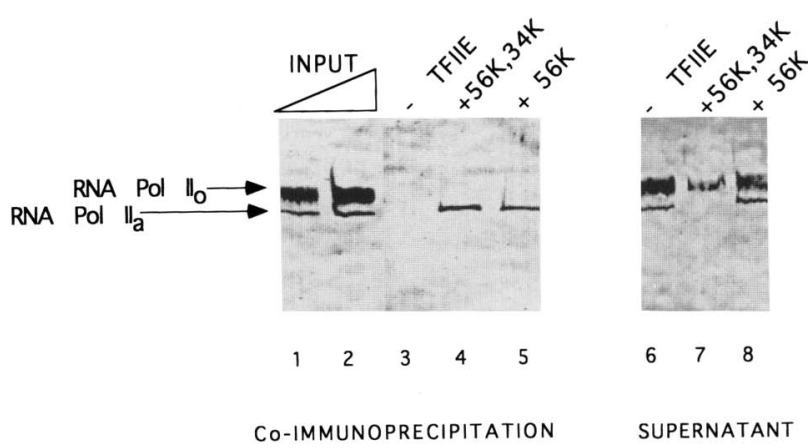

Figure 1. TFIIE preferentially binds the nonphosphorylated form of RNA Pol II. A mixture of RNA Pol IIo and RNA Pol IIa (lanes 1,2$)$ was used in coimmunoprecipitation experiments. The binding of RNA Pol II to TFIIE was analyzed using antiTFIIE-56 antiserum alone (lane 3 ) and in the presence of the TFIIE subunits: TFIIE-56 (lane 4) TFIIE-56, and TFIIE-34 (lane 5). The corresponding supernatants of the immunoprecipitations are shown in lanes 6,7 , and 8 respectively. The presence of the large subunit of RNA Pol II was detected by Western blot using a monoclonal antibody specific for the carboxy-terminal domain. 
lated form of the enzyme can be coimmunoprecipitated (data not shown), suggesting that in the absence of RNA Pol IIa, TFIIE can weakly recognize RNA Pol IIo consistent with the observation that RNA Pol IIo can be inefficiently assembled into the transcription complex (Lu et al. 1991). Our results suggest that in addition to preferentially recognizing the nonphosphorylated form of RNA Pol II, TFIIE may as a consequence be routinely recruited to the preinitiation complex even though it may not be required until after the initiation of the first phosphodiester bond.

\section{TFIIE interacts with TFIIF}

Because TFIIF is thought to recruit RNA Pol Ila to the promoter (Flores et al. 1991) and enters the transcription complex with RNA Pol IIa, we were interested in determining whether TFIIE can also interact directly with TFIIF. We generated two specific affinity resins using polyclonal antibodies against the subunits of TFIIE and protein A-Sepharose beads. Both resins nonspecifically retain a small amount of the TFIF subunits, RAP30 and RAP74, in the absence of TFIIE subunits (Fig. 2, lanes 1 and 2 for $\alpha$-TFIIE-56 resin and lane 9 for $\alpha$-TFIIE-34 resin). The addition of purified recombinant TFIIE- 56 to $\alpha$-TFIIE- 56 resin results in little or no binding of RAP30 above the background level (Fig. 2, lane 3), but leads to a

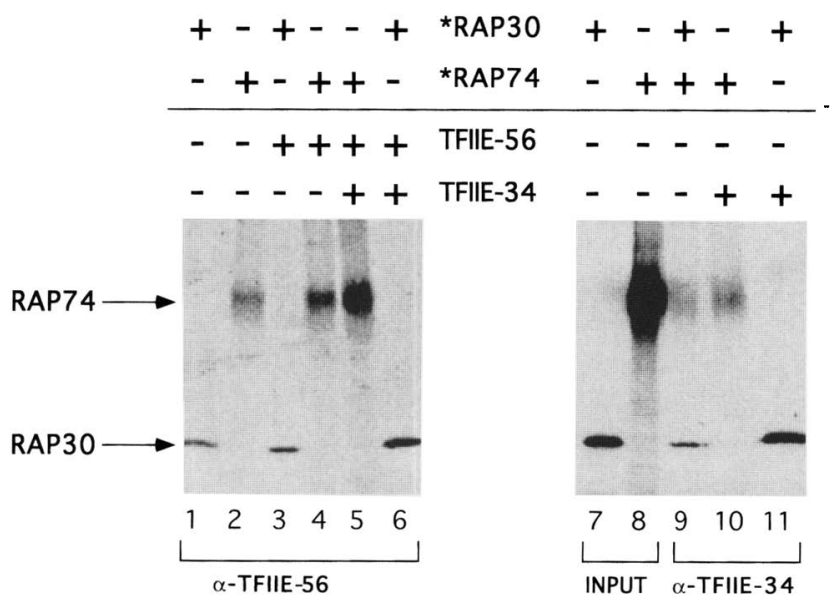

Figure 2. TFIIE Interacts with TFIIF. Radiolabeled subunits of TFIIF, "RAP30, and *RAP74 were tested for their ability to be coimmunoprecipitated with TFIIE subunits. Lanes 7 and 8 show the input proteins *RAP30 and *RAP74, respectively. Proteins were tested for nonspecific binding on control resins consisting of protein A-Sepharose and $\alpha$-TFIIE-56 antiserum (lanes 1,2) and $\alpha$-TFIIE-3 antiserum (lane 9) in the absence of added recombinant TFIIE subunits. The addition of TFIIE- 56 to $\alpha$-TFIIE-56 immuno-affinity resin results in little or no specific retention of ${ }^{*}$ RAP30 (lane 3) but results in a significant retention of * RAP74 (lane 4). The addition of TFIIE-34 in the presence of TFIIE-56 results in an even greater amount of *RAP74 (lane 5) and ${ }^{*}$ RAP30 (lane 6) retained by $\alpha$-TFIIE-56 immuno-affinity resin. In the presence of TFIIE-34, $\alpha$-TFIIE-34 immuno-affinity resin retains no *RAP74 above background (lane 10) but specifically retains a significant level of *RAP30 above background (lane 11). modest but significant amount of specific RAP74 binding (Fig. 2, lane 4). The addition of purified recombinant TFIIE-34 to the TFIIE-56 resin results in a marked increase in the amounts of RAP74 (Fig. 2, lane 5) and RAP30 (Fig. 2, lane 6) bound by the resin relative to background levels (Fig. 2, lanes 2 and 1, respectively). A similar analysis with $\alpha$-TFIIE- 34 resin results in a specific retention of RAP30 (Fig. 2, lane 11) above nonspecific binding (Fig. 2, lane 9), although there is little or no specific binding of RAP74 (Fig. 2, lane 10) by TFIIE-34 above background (Fig. 2, lane 9). Interestingly, it appears that under the conditions of our assay, RAP30 is the subunit of TFIIF bound preferentially by TFIIE-34 (Fig. 2, lane 11) and RAP74 is the subunit bound preferentially by TFIIE56 (Fig. 2, lane 4). Furthermore, TFIIE-56 specifically retains an increased amount of RAP74 in the presence of TFIIE-34, suggesting that TFIIE-34 is required for stable interactions between TFIIE and TFIIF. In light of our observation that TFIIE-56 can stably associate with RNA Pol IIa in the absence of TFIIE-34, it is possible that TFIIE-34-TFIIF interactions function to help stabilize the complex. This may explain the observation that TFIIE-56-RNA Pol IIa interactions are not sufficient for the formation of a stable transcription complex during gel mobility-shift assays (Peterson et al. 1991); the requirement for an equimolar amount of TFIIE-34 suggests that TFIIE-34-mediated binding of TFIIF may contribute to the stable association of the TFIIE heterotetramer with the transcription complex.

\section{TFIIE interacts with TBP}

Given the selective interaction of TFIIE with the nonphosphorylated form of RNA Pol II, and earlier studies that report a direct interaction between TBP and the nonphosphorylated CTD (Usheva et al. 1992), we considered the possibility that TFIIE may also interact with TBP. To test this idea, we generated a resin containing an immobilized glutathione-S-transferase-TFIIE-56 (GST56) fusion protein and analyzed the ability of this protein-affinity resin to specifically retain $\left[{ }^{35} \mathrm{~S}\right]$ methioninelabeled in vitro-translated TBP. Several other subunits of TFIID including $\mathrm{dTAF}_{\mathrm{II}} 30 \alpha$ and $\mathrm{dTAF}_{\mathrm{II}} 30 \beta$, as well as the largest subunit of the basal transcription factor TFIIA were also tested as controls for specificity of binding. Under the conditions of our assay, TBP was the only protein specifically retained by GST- 56 beads (Fig. 3A, lane 8). In contrast, TFIIA, $\mathrm{dTAF}_{\text {II }} 30 \alpha$, and $\mathrm{dTAF}_{\mathrm{II}} 30 \beta$ (lanes 6 and 7 , respectively), recombinant proteins shown to bind other partners in similar assays /Yokomori et al. 1993a,b), failed to bind, suggesting that the observed interaction between TBP and TFIIE-56 is specific.

If the interaction between in vitro-translated radiolabeled TBP and GST-56 is significant, we would expect that TBP-TFIIE-56 binding should also occur when purified recombinant TBP is tested. As predicted, GST-56 affinity resin bound TBP (Fig. 3B, lane 3) whereas GST control resin failed to bind TBP (Fig. 3B, lane 2). In further experiments, not shown here, several additional af- 


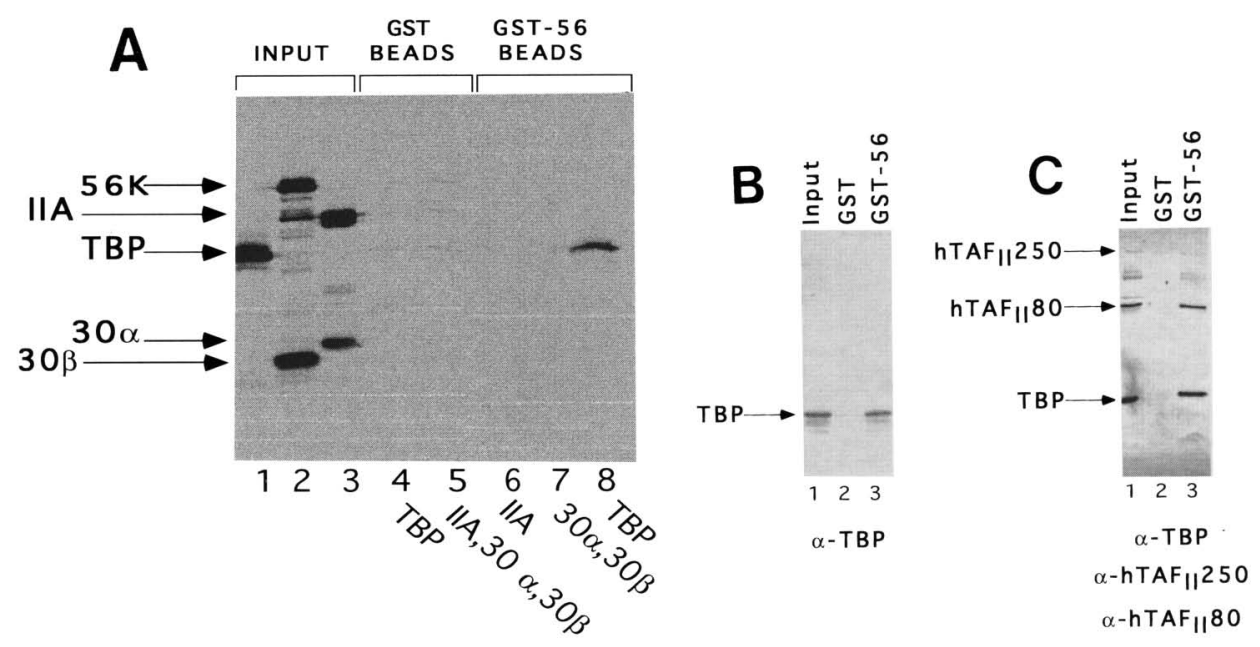

Figure 3. TFIIE-56 interacts with TBP and TFIID. (A) Radiolabeled RNA Pol II-specific transcription factors were tested for their ability to bind to a GST-56 fusion protein immobilized on glutathione beads in $0.15 \mathrm{M} \mathrm{KCl}$ binding buffer. Lanes $1-3$ show the input of TBP, $\mathrm{dTAF}_{\mathrm{II}} 30 \beta, \mathrm{dTAF}_{\mathrm{II}} 30 \alpha$, and the large subunit of TFIIA. Proteins were tested for nonspecific binding on a control GST resin (lanes 4, 5; proteins tested as indicated) and for TFIIE-56-specific binding on GST-56 resin (lanes 6-8) as indicated. (B) Bacterially expressed purified human TBP was assayed for its ability to bind GST-56 resin in $0.1 \mathrm{M} \mathrm{KCl}$ binding buffer, and the bound material was visualized by Western blot analysis using a monoclonal antibody to TBP. Lane 1 shows the TBP input. TBP is specifically retained by GST-56 resin (lane 3) but not by GST control resin (lane 2). (C) A partially purified TFIID fraction was tested for the ability to bind GST-56 resin in $0.1 \mathrm{M} \mathrm{KCl}$ binding buffer, and the bound material was visualized using a cocktail of antibodies specific for TBP, $\mathrm{hTAF}_{\mathrm{II}} 80$, and $\mathrm{hTAF}_{\mathrm{II}} 250$. Lane 1 shows the input TFIID fraction. GST-56 specifically retains TBP, hTAF $\mathrm{II}_{\mathrm{II}} 80$, and hTAF 250 (lane 3 ) but control GST resin does not (lane 2).

finity resins, including GST-TBP, GST-carboxy-terminal TBP, and TFIIE-56 immuno-affinity resins, were generated to analyze TFIIE-56-TBP binding. In all cases, stable TFIIE-56-TBP binding was observed, supporting the notion that TFIIE-56 and TBP interact, and do so via the conserved carboxy-terminal domain of TBP.

Because it is likely that TBP seldom exists as a single subunit in vivo, we were also interested in determining whether TFIIE- 56 could interact with the native TFIID complex. Using GST-56 resin and a phosphocellulose fraction containing native TFIID, we found that the GST-56 resin was competent for binding to the TFIID complex, as determined by Western blotting using a cocktail of antibodies that recognizes TBP, as well as two other subunits of TFIID, hTAF ${ }_{\text {II }} 80$ and hTAF II $_{\text {II }} 250$ (Fig. $3 \mathrm{C}$, lane 3). This result not only demonstrates that TFIIE can bind native TFIID, but also that this interaction results in a TFIIE-TFIID complex that is stable for in vitro isolation. These findings, taken together with the RNA Pol II data, indicate that TFIIE participates in interactions with both RNA Pol II and with TBP, a protein known also to bind to the nonphosphorylated CTD of RNA Pol II. It therefore became of interest to determine whether TFIIE could also interact directly with and thereby recruit TFIIH, a factor known to bind after TFIIE complex assembly (Flores et al. 1992) and reported to possess RNA Pol II CTD kinase activity (Lu et al. 1992).

\section{TFIIE interacts with TFIIH and ERCC-3}

The multisubunit TFIIH fraction has been shown to phosphorylate the CTD of RNA Pol II, and TFIIE can stimulate the kinase activity of TFIIH ( $\mathrm{Lu}$ et al. 1992). We therefore examined whether or not TFIIE could bind stably to TFIIH. GST-56 resin was prebound with TFIIE34 to generate a matrix containing both subunits of TFIIE. Using a partially purified fraction that contains TFIIH transcriptional activity, we tested the ability of the TFIIE resin to interact with TFIIH. We identified the bound species by Western blot analysis using antibodies to the cloned 62-kD subunit of TFIIH, p62 (Fischer et al. 1992). As shown in Figure 4, TFIIE resin retains an increasing amount of TFIIH p62 as the amount of TFIIH fraction is increased (lanes 3-5), and there is a corresponding depletion of TFIIH p62 from the supernatant. These data suggest that TFIIE can bind specifically and directly to one or more subunits of TFIIH.

Recently, ERCC-3, a protein known to be involved in DNA repair (Weeda et al. 1990), has been reported to be a subunit of the TFIIH complex (Schaeffer et al. 1993). We examined whether or not TFIIE stably interacts with ERCC-3 using a maltose-binding protein (MBP)-ERCC-3 fusion product linked to amylose beads (MBP-ERCC-3 resin). We found that the MBP-ERCC-3 resin could retain both the large and small subunits of TFIIE (Fig. 5, lanes 4-6), whereas control MBP could not (Fig. 5, lane 3). In addition, MBP-ERCC-3 resin does not retain a control protein, TFIIA (Fig. 5, lane 9), suggesting that the interaction of TFIIE with ERCC-3 is specific. These results indicate that TFIIE interacts with ERCC-3 through protein-protein contacts.

Having established that TFIIE can interact with TFIIH, we then tested the ability of TFIIE to recruit the intact multiprotein TFIIH complex. Specifically, we asked 


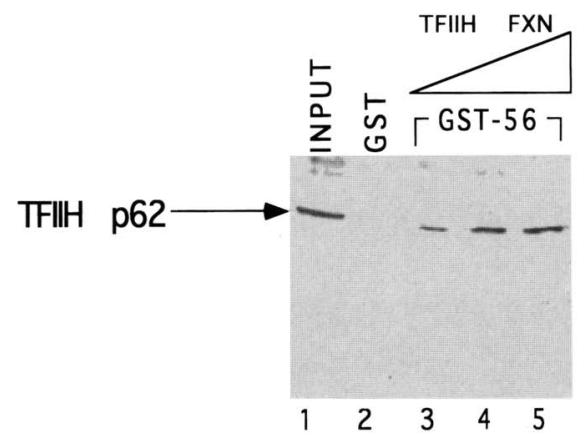

BEADS

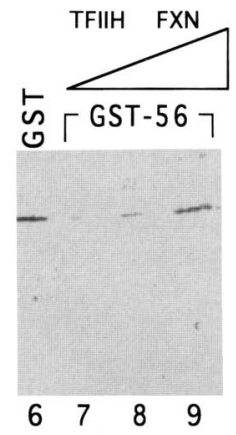

SUPERNATANT

Figure 4. TFIIE Interacts with TFIIH. A partially purified TFIIH preparation (lane 1 ) was tested for the ability to bind GST-56 resin in $0.1 \mathrm{~m}$ binding buffer and was analyzed by Western blot using antibodies against the p62 subunit of TFIIH. Incubation of the partially purified TFIIH fraction with GST control resin resulted in no retention of p62 in the beads (lane 2). Instead, p62 remained in the supernatant in its intact form (lane 6). In contrast, GST-56 resin efficiently retained p62 (lanes 3-5) such that a corresponding depletion of the supernatant was observed (lanes 7-9).

whether one of the TFIIE subunits could affinity isolate functional TFIIH. A partially purified TFIIH preparation was applied to the GST-56 affinity resin under relatively

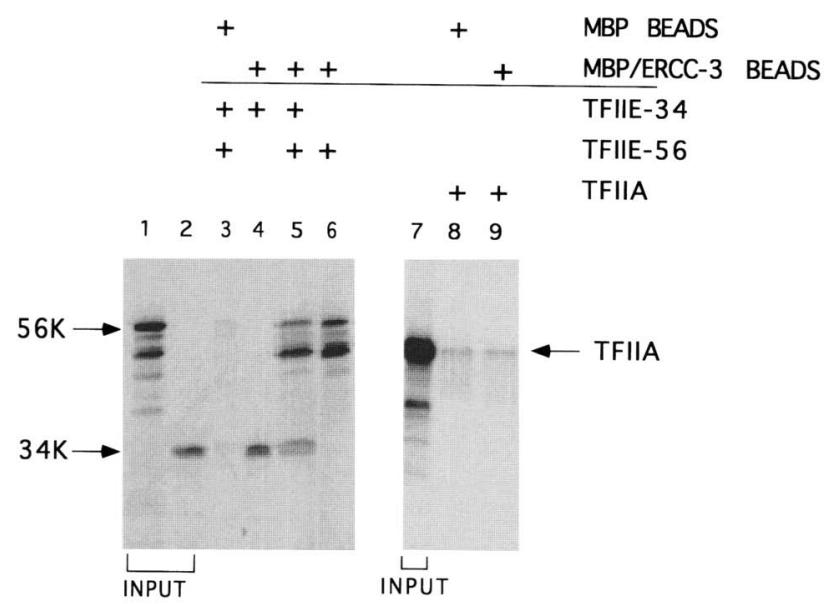

Figure 5. TFIIE interacts with ERCC-3. Radioactive TFIIE subunits were tested for the ability to bind a MBP-ERCC-3 fusion protein immobilized on amylose beads and in $0.1 \mathrm{M}$ binding buffer. Lanes 1 and 2 show input TFIIE-56 and TFIIE-34, respectively. Arrows indicate positions of full-length products. Control MBP beads did not retain either subunit of TFIIE (lane 3). In contrast, MBP-ERCC- 3 resin retained both the TFIIE-56 and TFIIE-34 subunits of TFIIE when they were tested independently (lanes 6 and 4, respectively) or together (lane 5). Radioactive TFIIA large subunit, a protein known to bind other proteins in similar assays (Yokomori et al. 1993a), did not bind to either the control MBP or MBP/ERCC-3 resins /lanes 8 and 9, respectively). low ionic conditions. After repeated washing to remove unbound material, we eluted the beads with high salt. We observed TFIIH p62 in the $0.5 \mathrm{M}$ eluate from TFIIE resin (Fig. 6A, lane 4) but not from control GST resin (Fig. 6A, lane 2). Importantly, activity eluted from the GST-56 resin was able to restore transcriptional activity to a reconstituted reaction dependent on added TFIIH (Fig. 6B, lanes 9,10). Material eluted from the control resin had no TFIIH activity (Fig. 6B, lanes 5,6 ). The reconstitution of transcription by the fraction eluted from the GST-56 resin suggests that the bound material contains an active form of TFIIH and that the interactions between TFIIE and TFIIH observed in vitro may reflect the recruitment of TFIIH to the transcription complex by TFIIE.

\section{Discussion}

Our efforts to define the role of TFIIE in transcription have focused on a systematic analysis of interactions of
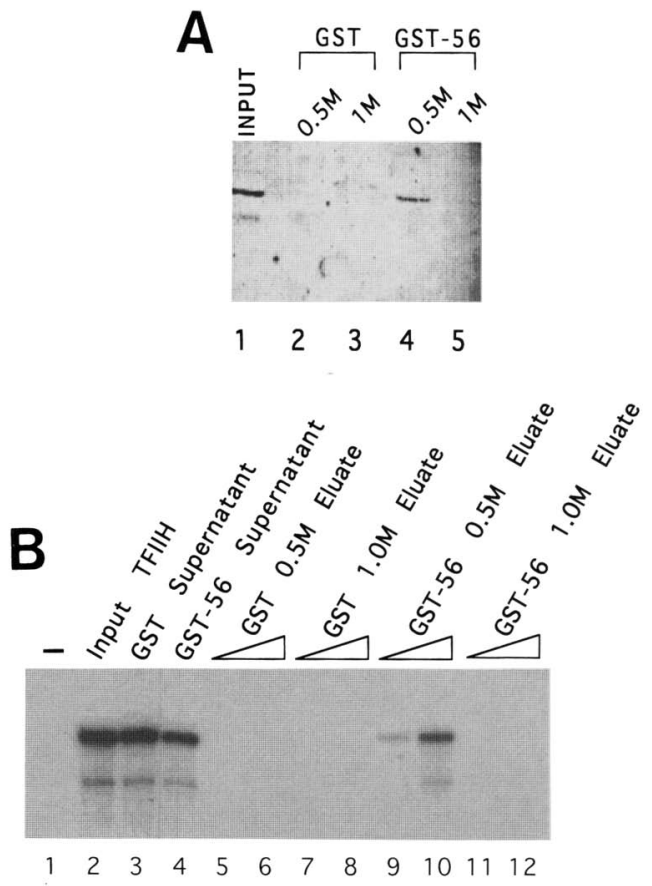

Figure 6. TFIIE-56 resin can affinity purify a transcriptionally active TFIIH complex. (A) A partially purified TFIIH fraction was applied to control GST or GST-56 resin under low ionic strength conditions $(0.1 \mathrm{M}$ binding buffer) and washed extensively. Western blot analysis using TFIIH p62 antibodies revealed that the $0.5 \mathrm{M} \mathrm{KCl}$ elution from GST-56 resin contained TFIIH p62 (lane 4), where an equivalent elution from GST control resin did not (lane 2). (B) The $0.5 \mathrm{M}$ eluate from the GST-56 resin contains TFIIH transcriptional activity. A reconstituted HeLa transcription system that was absolutely dependent on TFIIH (lanes 1,2) was used to test the GST and GST-56 resin eluates for TFIIH activity. The $0.5 \mathrm{M}$ eluate from the GST-56 resin specifically contained TFIIH activity (lanes 9,10 ), whereas both the $0.5 \mathrm{M}$ and $1.0 \mathrm{M}$ eluates from the control GST resin (lane $5-8$ ) as well as the $1.0 \mathrm{M}$ eluate from the GST-56 resin (lanes 11,12 ) did not contain TFIIH activity. 
TFIIE with known components of the basal transcription machinery and have revealed that this basal factor participates in several potentially important interactions with many of the components of the RNA Pol II transcription complex. Significantly, TFIIE binds preferentially to the nonphosphorylated form of RNA Pol II, despite the presence of a molar excess of the phosphorylated form. TFIIE also associates with TFIID and binds selectively to the multisubunit TFIIH complex. The interaction between TFIIE and TFIIH is sufficiently specific and robust in vitro to allow the purification of a transcriptionally active TFIIH complex. Additional binding studies reveal that TFIIE participates in protein-protein contacts with TFIIF, and with ERCC-3, a DNA repair helicase reported to be a subunit of TFIIH (Schaeffer et al. 1993). These binding studies support the notion that TFIIE is an integral component of the transcription complex responsible for orchestrating or directing multiple interactions, and that these interactions may play a role in recruiting essential transcription factors such as TFIIH. Though these data indicate that specific interactions between components of the transcription complex can occur in vitro, further experimentation is required to assess any functional relevance of these interactions in vivo.

Recent in vitro studies have demonstrated that TFIIE is not required for transcription of certain promoters on supercoiled templates while it is required for transcription from the same promoters on linear templates (Parvin and Sharp 1993). However, abortive initiation as- says that detect open promoter complexes capable of cycling trinucleotide transcripts, a hallmark of the initiation step of transcription, have revealed that TFIIE is not required for initiation of transcription even from linear templates (Goodrich and Tjian 1994). Furthermore, analysis of ternary elongation complexes suggests that TFIIE is not associated with the elongating RNA Pol II complex (D. Reinberg, pers. comm.). Our efforts to define the function of TFIIE have revealed some intriguing results establishing that TFIIE interacts with multiple components of the transcription machinery. Our results, taken together with recent transcription experiments that dissect some of the discrete steps during transcription (i.e., initiation, promoter clearance, and elongation) suggest a model for the assembly, recruitment and subsequent dynamic alteration that must occur during the transition from initiation to elongation. A model for the potential participation of TFIIE during transcription is shown in Figure 7.

First, the recruitment of TFIIE to the promoter follows the simultaneous association of TFIIF and the nonphosphorylated form of RNA Pol II (IIa) to the TFIID/TFIIB complex. We envision that TFIIE enters into the transcription complex through multiple interactions with several components already assembled at the promoter including RNA Pol II, TBP, and TFIIF. One of the primary interactions may be between TFIIE and RNA Pol IIa, the form of the enzyme recruited to the promoter by TFIIF. The observed selectivity of TFIIE for the (nonphosphorylated) RNA Pol Ila suggests that TFIIE may
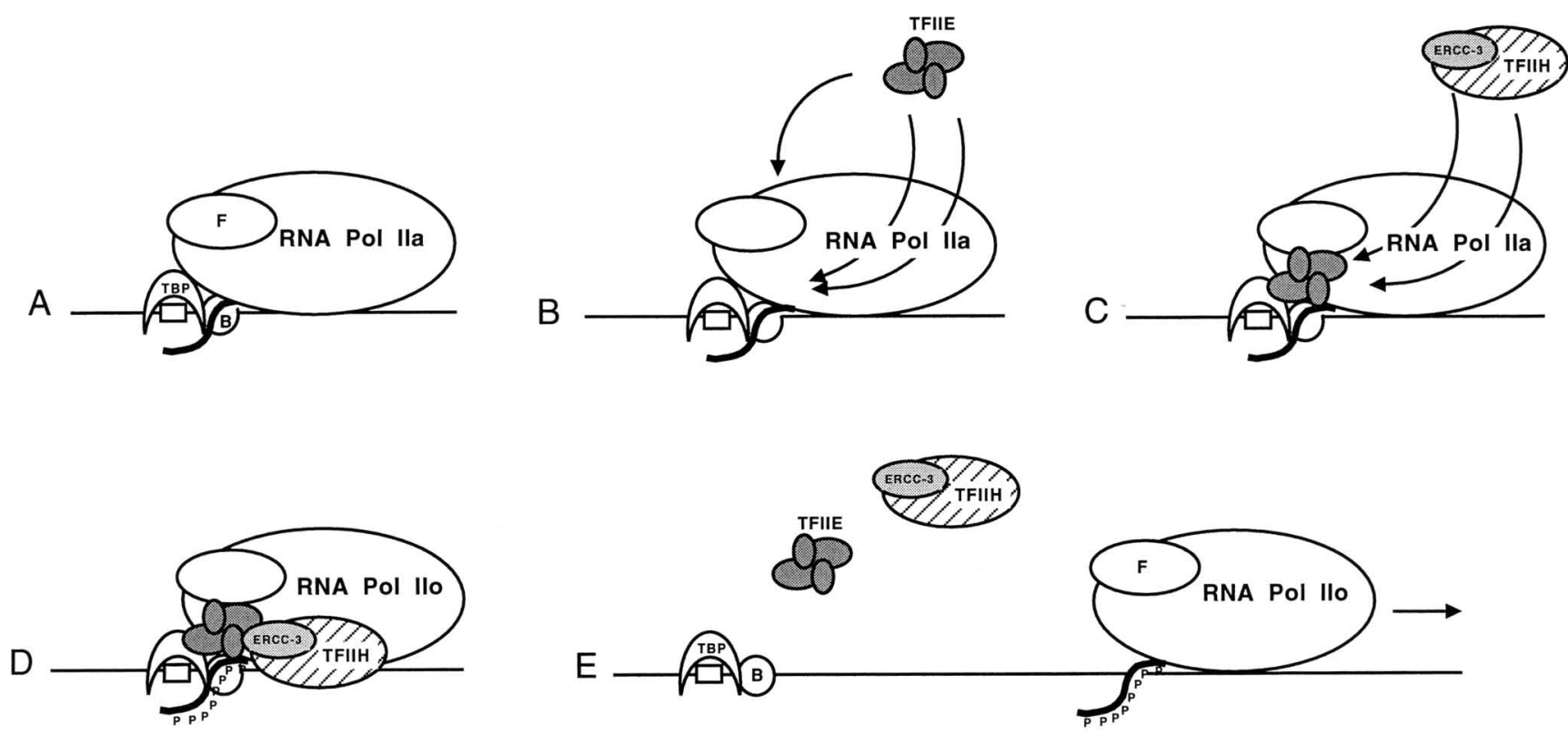

Figure 7. A model for the role of TFIIE in the process of promoter clearance. $(A)$ The active initiation complex (for the purposes of simplicity, TFIIA, and components of TFIID other than TBP have been omitted). (B) Assembly of TFIIE into the complex via binding of RNA Pol IIa, TBP, and TFIIF. (C) TFIIE recruits TFIIH and ERCC-3 by direct interactions with both TFIIE-34 and TFIIE-56 subunits. (D) Association of TFIIH with the transcription complex allows for the phosphorylation of the CTD of RNA Pol II. (E) Promoter clearance occurs, releasing TBP, TFIB, TFIIE, and TFIIH and triggering the elongation mode of transcription by RNA Pol IIo. 
bind the nonphosphorylated CTD directly as a mechanism for discriminating between the two forms. However, our preliminary studies did not reveal any stable interaction between the CTD and TFIIE subunits (data not shown). Alternatively, TFIIE may interact with other domains or subunits of RNA Pol II that are conformationally sensitive to the phosphorylation state of the CTD. Because the nonphosphorylated RNA Pol II is bound by both TBP and TFIIE, the binding of TFIIE may serve to stabilize the partially assembled transcription complex and also mediate the formation of an interface for TFIIH binding. Further evidence for a role for TFIIE in complex stabilization is provided by preliminary experiments using TFIIE affinity resins showing that TFIIE also interacts with TFIIB. We believe these interactions to be specific and primarily mediated by TFIIE-34, the small subunit of TFIIE (data not shown).

Previous studies have revealed that TFIIH does not bind a partially formed transcription complex in the absence of TFIIE as measured by gel mobility-shift assays (Flores et al. 1992). Our results now establish that TFIIE most likely interacts directly with components of TFIIH and can stably bind to an intact and functional TFIIH complex. These specific and possibly obligatory proteinprotein contacts between TFIIE and TFIIH are likely to recruit TFIIH to the transcription complex containing the nonphosphorylated form of RNA Pol II. This recruitment of TFIIH by TFIIE may in turn target TFIIH to the CTD, thus mediating the subsequent phosphorylation of the CTD by the kinase activity associated with TFIIH. The change in the phosphorylation state of RNA Pol II may in turn signal the release of TBP (Usheva et al. 1992; Drapkin et al. 1993), and (because it does not bind the phosphorylated form) TFIIE from RNA Pol II, thus altering the assembly and poising the complex for movement from the open promoter structure into the elongation mode of transcription. Although TFIIH has been shown to phosphorylate the CTD in the absence of the other components of the transcription complex in vitro, kinase activity is greatly stimulated by assembly of a preinitiation complex including TFIIE. More specifically, the addition of TFIIE has been reported to stimulate the kinase activity of TFIIH both qualitatively /conferring the efficient use of $\left[\gamma^{-32} \mathrm{P}\right]$ ATP as a substrate) and quantitatively (contributing to a fourfold increase in phosphorylation of RNA Pol II) (Lu et al. 1992). Therefore, TFIIE may not only recruit TFIIH, but target it directly to the CTD, and/or induce a conformational change in TFIIH that facilitates its kinase activity. We propose that recruitment of TFIIH by TFIIE is required to enable kinase and/ or helicase functions in order to trigger the promoter clearance step of transcription.

Our experiments also reveal an interaction between TFIIE and ERCC-3, a DNA-repair protein reported to be a subunit of TFIIH. Recently, factor $b$, the yeast equivalent to TFIIH, has been reported to be comprised primarily of DNA repair proteins (Feaver et al. 1993). The association of TFIIE, TFIIH, and DNA repair proteins suggests that these factors may function to couple transcription and DNA repair. Several reports indicate that the template strand of actively transcribed genes is repaired more efficiently than the nontemplate strand, and considerable data support the proposed coordination of these two processes in both eukaryotes (Bohr et al. 1985; Mellon et al. 1986) and prokaryotes (Mellon and Hanawalt 1989; Selby and Sancar 1993). Although the coupling of transcription and DNA repair remains less clear in eukaryotes, the ERCC-6, ERCC-3, and ERCC-2 gene products have been implicated in the process (Wauthier et al. 1990; Lommel and Hanawalt 1991). Interestingly, the association of ERCC-3 with TFIIE and TFIIH implies a recruitment of such DNA repair proteins during the assembly of the transcription complex.

The association of DNA repair proteins with TFIIE/ TFIIH raises the following question: Why are DNA repair components coupled to transcription factors involved in early events during transcription? The interaction of DNA repair proteins with the transcriptional apparatus raises the possibility that TFIIE may participate in two distinct functions. It is possible that like RNA Pol IIa at the the initiation site which requires additional factors (TFIIE and TFIIH) to clear the promoter, RNA Pol II stalled at a DNA lesion may require the reassembly of additional factors to repair the lesion and continue the process of transcription. In this model, the RNA Pol II stalled at a lesion would be recognized by TFIIE which, perhaps via ERCC-3, recruits TFIIH and the associated DNA repair proteins to the lesion. Furthermore, this model proposes that stalled RNA Pol II would exist in the dephosphorylated form, the species that is able to efficiently bind TFIIE. Dephosphorylation of RNA Pol II stalled at a template lesion could perhaps be generated through the action of an associated phosphatase that specifically recognizes a halted RNA Pol II complex. In support of this notion, perhaps, is the observation of an activity in HeLa cell extracts that has been shown to catalyze the dephosphorylation of the CTD (Chesnut et al. 1992), though the substrate specificity and characterization of this activity remain unclear. An example of a condition where re-recruitment of TFIIE/ TFIIH may be required apparently exists at the Drosophila melanogaster HSP70 promoter, where analysis has revealed that the RNA Pol II halted at the pause site (+20 to +40 relative to the start of transcription), is in the nonphosphorylated state in vivo (T. O'Brien and J. Lis, pers. comm.). Whether the nonphosphorylated RNA Pol II in these experiments is a result of a dephosphorylation event or reflects a condition where RNA Pol II was not initially phosphorylated prior to transcription is not known. Because the available data suggest that in vivo, genes that are actively transcribed are associated specifically with RNA Pol IIo (Weeks et al. 1993) and have not yet been shown to be associated with RNA Pol Ha under any experimental conditions, it is possible that a rephosphorylation event must occur before the stalled complex can continue elongation. A re-recruitment of TFIIE and TFIIH may be required before the halted RNA Pol II, be it at a pause site or a DNA lesion, can resume elongation of the transcript. The formation of an assembled complex containing TFIIE may therefore lead to either initi- 
ation or repair, depending on the status of the template bound by RNA Pol II. Assembly of such a "bifunctional" transcription complex provides flexibility in supplying a modular set of factors that can participate in transcription or DNA repair. Although it is currently unknown whether an RNA Pol II complex stalled at a DNA lesion resumes elongation from the stall site, or whether the entire complex dissociates and transcription begins anew, our observations provide a basis for further experimentation to address both possibilities.

Although it is possible that the role of TFIIE in transcription is primarily to recruit $\mathrm{TFIIH}$, an analysis of the primary sequence of the TFIIE subunits has revealed computer-predicted similarities to several interesting structural features suggesting that TFIIE may serve additional functions. For example, the observation that TFIIE possesses structural similarities to the bacterial DNA repair protein UvrA (Peterson et al. 1991) is suggestive of a direct involvement of TFIIE in the process of DNA repair. In addition to UvrA similarity, TFIIE has been predicted to have a zinc coordination domain, a nucleotide-binding site, and a region similar to the catalytic domain of several protein kinases. The precise mechanism by which these predicted features of TFIIE may contribute to the processes of transcription and DNA repair is currently under investigation using various strategies employing mutagenesis. The analysis of recombinant TFIIE subunits carrying mutations targeted to computer-predicted structural features in reconstituted transcription, affinity interaction, and DNA repair assays is expected to facilitate the characterization of discrete domains that may be responsible for governing the activities of TFIIE in both transcription and transcription-coupled DNA repair.

\section{Materials and methods}

\section{Generation of polyclonal antibodies against TFIIE subunits}

Polyclonal antisera against TFIIE-56 and TFIIE-34 were raised in rabbits using Escherichia coli-expressed recombinant proteins, which were purified as described previously (Peterson et al. 1991). Antigens were prepared using Freund's complete and incomplete adjuvant, and injections were done subcutaneously. Antisera were tested for reactivity and specificity in Western assays.

\section{Protein-protein interaction assays}

Coimmunoprecipitation assays were performed as described previously (Yokomori et al. 1993a) using $2 \mu \mathrm{l}$ of $\alpha$-TFIIE-56 or $\alpha$-TFIIE-34 antiserum, where indicated, and $0.1 \mathrm{M} \mathrm{KCl} / \mathrm{HEMG}-$ binding buffer containing $0.01 \% \mathrm{NP}-40$ and $0.5 \mathrm{mM}$ DTT.

RNA Pol II containing a mixture of RNA Pol IIa and RNA Pol IIo forms was purified as described previously (Lu et al. 1991), and $\sim 250-400 \mathrm{ng}$ was used per assay. Where indicated, 300 and $350 \mathrm{ng}$ of recombinant TFIIE-56 and TFIIE-34 were used, respectively, in assays. Coimmunoprecipitations were done in binding buffer described above, and beads were washed extensively and boiled in SDS-PAGE sample buffer prior to loading on $6 \%$ gels. Proteins were visualized after transfer to nitrocellulose using a
1:5000 dilution of a monoclonal antibody specific for the large subunit of RNA Pol II obtained from Promega.

TFIIF interaction assays were performed as described for RNA Pol II interaction assays using immunoaffinity resins for TFIIE34 and TFIIE-56, with the following modifications. RAP30 and RAP74 were radiolabeled using the TNT T7 Coupled Reticulocyte Translation system available from Promega. Recombinant TFIIE subunits were prebound to the appropriate immunoaffinity resin, the beads were washed extensively, and radioactive TFIIF subunits were added as indicated in the legend to Figure 2. Beads were washed extensively after a $2 \mathrm{hr}$ incubation, resuspended in SDS-PAGE sample buffer, and run on a $10 \%$ SDSPAGE gel prior to visualization via autoradiography.

TBP coimmunoprecipitation assays were performed using anti-TFIIE-56 antiserum, $300 \mathrm{ng}$ of TFIIE-56, and $150 \mathrm{ng}$ of recombinant human TBP purified from $E$. coli as described previously (Peterson et al. 1990) and visualized by Western analysis using a monoclonal antibody specific for TBP (Lobo et al. 1992), kindly provided by Nouria Hernandez (Cold Spring Harbor Laboratory, Cold Spring Harbor, NY).

TBP interaction assays using glutathione-agarose beads were performed as described previously (Goodrich et al. 1993), with the following modifications. $\left[{ }^{35} \mathrm{~S}\right]$ Methionine labeling of proteins was performed using the TNT T7 Coupled Reticulocyte Translation system available from Promega. Proteins were incubated with the GST and GST-chimera beads for $2 \mathrm{hr}$ at $4^{\circ} \mathrm{C}$. The total amount of GST fusion protein was $\sim 1.5 \mu \mathrm{g}$, and the concentrations of $\mathrm{KCl}$ used are indicated in the legend to Figure 3 . Where radioactive proteins were used, SDS-PAGE gels were dried and exposed to film.

TFIIH interaction assays were performed using beads containing $0.5 \mu \mathrm{g}$ of GST-56 fusion protein and $40 \mu \mathrm{l}$ of a partially purified TFIIH fraction (Flores et al. 1992) in $0.1 \mathrm{M} \mathrm{KCl} \mathrm{HEMG}$ binding buffer. The beads were washed extensively and resuspended in SDS-PAGE sample buffer prior to boiling and loading onto a $10 \%$ SDS-polyacrylamide gel. The bound material was visualized by Western blot using 1:1000 dilution of antibodies against the p62 subunit of TFIIH.

TFIIH interaction/elution assays were performed using beads containing $0.7 \mu \mathrm{g}$ of GST-56 fusion protein and $100 \mu \mathrm{l}$ of a partially purified phenyl-Superose TFIIH fraction (Flores et al. 1992). The incubation was performed at $4^{\circ} \mathrm{C}$ for $1.5 \mathrm{hr}$ in $\mathrm{HEMG}$ binding buffer containing $0.1 \mathrm{M} \mathrm{KCl}$ and $4 \mathrm{mM} \mathrm{MgCl}_{2}$. The beads were washed extensively and eluted for $45 \mathrm{~min}$ using $100 \mu \mathrm{l}$ of binding buffer containing $0.5 \mathrm{M} \mathrm{KCl}$, and subsequently with 100 $\mu \mathrm{l}$ buffer containing $1.0 \mathrm{M} \mathrm{KCl}$. Eluted material was dialyzed immediately into $0.1 \mathrm{~m}$ binding buffer for $3 \mathrm{hr}$ prior to transcription assay analysis. Eluted material was also resolved on a $10 \%$ SDS-polyacrylamide gel and visualized by Western blot using 1:1000 dilution of antibodies against the p62 subunit of TFIIH.

Interaction assays using amylose beads available from $\mathrm{New}$ England Biolabs were performed under conditions described for glutathione-agarose beads, with the following modification. The MBP-ERCC-3 fusion protein concentration was $\sim 3 \mu \mathrm{g}$ per assay and $0.15 \mathrm{M} \mathrm{KCl} \mathrm{HEMG-binding} \mathrm{buffer} \mathrm{was} \mathrm{used.}\left[{ }^{35} \mathrm{~S}\right] \mathrm{Me}$ thionine in vitro-translated TFIIE subunits were visualized via autoradiography.

All input lanes represent $10 \%$ of the amount of the indicated proteins tested in the assays.

\section{In vitro transcription}

In vitro transcription assays were performed in a HeLa transcription system reconstituted with recombinant human TBP, TFIIB, TFIIE, and TFIIF, highly purified RNA Pol II and a partially purified TFIIH fraction. The following recombinant pro- 
teins were expressed in E. coli and purified as described: TBP (Peterson et al. 1990); TFIIB (Ha et al. 1991); TFIIE-34 and TFIIE56 (Peterson et al. 1991); and RAP30 and RAP74 (Aso et al. 1992; Finkelstein et al. 1992). TFIIH was purifed as described (Flores et al. 1992) and was a kind gift from Lalo Flores. RNA Pol II was purified from HeLa cell nuclear pellets through the DEAE-5PW step as described (Lu et al. 1991). Transcription reactions were performed in a final volume of $25 \mu \mathrm{l}$ in buffer containing $20 \mathrm{~mm}$ Tris-Cl (pH 7.9), 10\% glycerol, 1 mM DTT, $4 \mathrm{mM} \mathrm{MgCl}_{2}, 50 \mathrm{~mm}$ $\mathrm{KCl}$, and $10 \mathrm{~mm}$ ammonium sulfate. Each reaction contained 20 $\mathrm{ng}$ of TBP, $20 \mathrm{ng}$ of TFIIB, $40 \mathrm{ng}$ of RAP30, $10 \mathrm{ng}$ of RAP74, 30 ng of TFIIE- $34,40 \mathrm{ng}$ of TFIIE-56, $125 \mathrm{ng}$ of RNA Pol II, and 200 ng of template DNA containing the adenovirus major late promoter $(-53$ to +10$)$ upstream of the G-less cassette (Sawadogo and Roeder 1985). The reactions did not contain 3'-O-Methyl GTP or RNase T1. Transcription factors were preincubated with DNA for $30 \mathrm{~min}$ at $30^{\circ} \mathrm{C}$. Nucleotides $(2 \mu \mathrm{l}$ of a $13 \times$ mixture) were added to give final concentrations of $500 \mu \mathrm{M}$ ATP, $500 \mu \mathrm{M}$ CTP, and $25 \mu \mathrm{M}\left[\alpha^{-32} \mathrm{P}\right] \mathrm{UTP}(5 \mu \mathrm{Ci})$. Transcription was allowed to proceed for $15 \mathrm{~min}$ at $30^{\circ} \mathrm{C}$. Reactions were stopped by the addition of $100 \mu \mathrm{l}$ of a solution containing $3.1 \mathrm{M}$ ammonium acetate, $10 \mu \mathrm{g}$ of carrier yeast RNA, and $15 \mu \mathrm{g}$ of proteinase $\mathrm{K}$. The samples were subjected to denaturing $6 \%$ PAGE after ethanol precipitation, and the transcripts were subsequently visualized by autoradiography.

\section{Acknowledgments}

We thank Danny Reinberg for support and helpful discussions, for communication of results before publication, and for the gift of TFIIH p62 antibodies. We are grateful to Lalo Flores for the partially purified TFIIH fractions and to Edith Wang for the native TFIID fraction. We also thank Sigi Ruppert for TFIIF in vitro expression constructs and for GST-TBP and GST-cTBP constructs, Aziz Sancar for permission to use the MBP-ERCC-3 fusion construct, and Kyoko Yokomori for TFIIA, $\operatorname{dTAF}_{\mathrm{II}^{-}}-30 \alpha$, and $\mathrm{dTAF}_{\mathrm{II}}-30 \beta$ in vitro expression constucts. We thank Tom $\mathrm{O}^{\prime}$ Brien for communication of results prior to publication and Tim Hoey, Lalo Flores, Greg Peterson, Grace Gill, Stig Hansen, Lucio Comai, and Gene Cutler for critical review of the manuscript. We also thank Gene Cutler and Karen Ronan for assistance with the figures. M.M. gratefully acknowledges Harry Lampiris, M.D., for his fundamental contributions to the completion of this work. J.A.G. is supported by a Damon RunyonWalter Winchell Cancer Research Fund Fellowship, (DRG1170). This work was supported, in part, by a grant from the National Institutes of Health to R.T.

The publication costs of this article were defrayed in part by payment of page charges. This article must therefore be hereby marked "advertisement" in accordance with 18 USC section 1734 solely to indicate this fact.

\section{References}

Aso, T., H.A. Vasavada, T. Kwaguchi, F.J. Germino, S. Ganguly, S. Kitajima, S.M. Weissman, and Y. Yasukochi. 1992. Characterization of cDNA for the large subunit of the transcription initiation factor TFIIF. Nature 355: 461-464.

Bohr, V.A., C.A. Smith, D.S. Okumoto, and P.C. Hanawalt. 1985. DNA repair in an active gene: Removal of pyrimidine dimers from the DHFR gene of CHO cells is much more efficient than in the genome overall. Cell 40: 359-369.

Buratowski, S., S. Hahn, L. Guarente, and P.A. Sharp. 1989. Five intermediate complexes in transcription initiation by RNA polymerase II. Cell 56: 549-561.
Chesnut, J.D., J.H. Stephens, and M.E. Dahmus. 1992. The interaction of RNA polymerase II with the adenovirus-2 major late promoter is precluded by phosphorylation of the C-terminal domain of subunit IIa. J. Biol. Chem. 267: 10500 10506.

Corden, J.L., D.L. Cadena, J.M.J. Ahearn, and M. Dahmus. 1985. A unique structure at the carboxyl terminus of the largest subunit of eukaryotic RNA polymerase II. Proc. Natl. Acad. Sci. 82: 7934-7938.

Drapkin, R., A. Merino, and D. Reinberg. 1993. Regulation of RNA polymerase II transcription. Curr. Opin. Cell Biol. 5: 469-476.

Dynlacht, B.D., T. Hoey, and R. Tiian. 1991. Isolation of coactivators associated with the TATA-binding protein that mediate transcriptional activation. Cell 66: 563-576.

Feaver, W., J.Q. Svejstrup, L. Bardwell, A.J. Bardwell, S. Buratowski, K.D.Gulyas, T.F. Donahue, E.C. Friedberg, and R.D. Kornberg. 1993. Cell 75: 1379-1387.

Finkelstein, A., C.F. Kostrub, J. Li, D.P. Chavez, B.Q. Wang, S.M. Fang, J. Greenblatt, and Z.F. Burton. 1992. A cDNA encoding RAP74, a general initiation factor for transcription by RNA polymerase II. Nature 355: 464-467.

Fischer, L., M. Gerard, C. Chalut, Y. Lutz, S. Humbert, M. Kanno, P. Chambon, and J.M. Egly. 1992. Cloning of the 62-kilodalton component of basic transcription factor BTF-2. Science 257: 1392-1395.

Flores, O., E. Maldonaldo, and D. Reinberg. 1989. Factors in volved in specific transcription by mammalian RNA polymerase II. Factors IIE and IIF independently interact with RNA polymerase II. I. Biol. Chem. 264: 8913-8921.

Flores, O., H. Lu, M. Killeen, J. Greenblatt, Z.F. Burton, and D. Reinberg. 1991. The small subunit of transcription factor IIF recruits RNA polymerase II into the preinitiation complex. Proc. Natl. Acad. Sci. 88: 9999-10003.

Flores, O., H. Lu, and D. Reinberg. 1992. Factors involved in specific transcription by mammalian RNA polymerase II. Identification and characterization of factor IIH. I. Biol. Chem. 267: 2786-2793.

Goodrich, J.A. and R. Tjian. 1994. Transcription factors IIE, IIH, and ATP hydrolysis direct promoter clearance by RNA polymerase II. Cell 77: (in press).

Goodrich, J.A., T. Hoey, C.J. Thut, A. Admon, and R. Tjian. 1993. Drosophila $\mathrm{TAF}_{\mathrm{II}} 40$ interacts with both a VP16 activation domain and the basal transcription factor TFIIB. Cell 75: $519-530$.

Ha, I., W.S. Lane, and D. Reinberg. 1991. Cloning of a human gene encoding the general transcription factor IIB. Nature 352: 689-695.

Inostroza, J., O. Flores, and D. Reinberg. 1991. Factors involved in specific transcription by mammalian RNA polymerase II: Purification and functional analysis of general transcription Factor IIE. J. Biol. Chem. 266: 9304-9308.

Laybourn, P.J. and M.E. Dahmus. 1990. Phosphorylation of RNA polymerase IIA occurs subsequent to interaction with the promoter and before the initiation of transcription. $I$. Biol. Chem. 265: 13165-13173.

Lobo, S.M., M. Tanaka, M.L. Sullivan, and N. Hernandez. 1992. A TBP complex essential for transcription from TATA-less but not TATA-containing RNA polymerase III promoters is part of the TFIIIB fraction. Cell 71: 1029-1040.

Lommel, L. and P.C. Hanawalt. 1991. The genetic defect in the chinese hamster ovary cell mutant UV61 permits moderate selective repair of cyclobutane pyrimidine dimers in an expressed gene. Mutat. Res: DNA Repair 255: 183-191.

Lu, H., O. Flores, R. Weinmann, and D. Reinberg. 1991. The nonphosphorylated form of RNA polymerase II preferen- 
tially associates with the preinitiation complex. Proc. Natl. Acad. Sci. 88: 10004-10008.

Lu, H., L. Zawel, L. Fisher, J.M. Egly, and D. Reinberg. 1992. Human general transcription factor IIH phosphorylates the C-terminal domain of RNA polymerase II. Nature 358: 641645.

Maldonado, E., I. Ha, P. Cortes, L. Weis, and D. Reinberg. 1990. Factors involved in specific transcription by mammalian RNA polymerase II: Role of transcription factors IIA, IID, and IIB during formation of a transcription-competent complex. Mol. Cell. Biol. 10: 6335-6347.

Mellon, I., and P.C. Hanawalt. 1989. Induction of the Escherichia coli lactose operon selectively increases repair of its transcribed strand. Nature 342: 95-98.

Mellon, I., V.A. Bohr, C.A. Smith, and P.C. Hanawalt. 1986. Preferential repair of an active gene in human cells. Proc. Natl. Acad. Sci. 83: 8878-8882.

Mitchell, P.J. and R. Tjian. 1989. Transcriptional regulation in mammalian cells by sequence-specific DNA binding proteins. Science 245: 371-378.

Parvin, J.D. and P. Sharp. 1993. DNA topology and a minimal set of basal factors for transcription by RNA polymerase II. Cell 73: 533-540.

Parvin, J.D., H.T.M. Timmers, and P. Sharp. 1992. Promoter specificity of basal factors. Cell 68: 1135-1144.

Peterson, M.G., N. Tanese, B.F. Pugh, and R. Tjian. 1990. Functional domains and upstream activation properties of cloned human TATA binding protein. Science 248: 1625-1630.

Peterson, M.G., J. Inostroza, M.E. Maxon, O. Flores, A. Admon, D. Reinberg, and R. Tjian. 1991. Structure and functional properties of human general transcription factor IIE. Nature 354: 369-373.

Reinberg, D. and R.G. Roeder. 1987a. Factors involved in specific transcription by mammalian RNA polymerase II. Purification and functional analysis of initiation factors IIB and IIE. I. Biol. Chem. 262: 3310-3321.

Sawadogo, M. and R. Roeder. 1985. Factors involved in specific transcription by human RNA polymerase II: Analysis by a rapid and quantitative in vitro assay. Proc. Natl. Acad. Sci. 82: 4394-4398.

Schaeffer, L., R. Roy, S. Humbert, V. Moncollin, W. Vermeulen, J.H.J. Hoeijmakers, P. Chambon, and J.M. Egly. 1993. DNA repair helicase: A component of BTF-2 (TFIIH) basic transcription factor. Science 260: 58-63.

Selby, C. and A. Sancar. 1993. Molecular mechanism of transcription-repair coupling. Science 260: 53-57.

Tanese, N., B.F. Pugh, and R. Tjian. 1991. Coactivators for a proline-rich activator purified from the multisubunit human TFIID complex. Genes \& Dev. 5: 2212-2224.

Tyree, C.M., C.P. George, L.M. Lira-Devito, S.L. Wampler, M.E. Dahmus, L. Zawel, and J.T. Kadonaga. 1993. Identification of a minimal set of proteins that is sufficient for accurate initiation of transcription by RNA polymerase II. Genes \& Dev. 7: 1254-1265.

Usheva, A., E. Maldonado, A. Goldring, H. Lu, C. Houbavi, D. Reinberg, and Y. Aloni. 1992. Specific interaction between the nonphosphorylated form of RNA polymerase II and the TATA-binding protein. Cell 69: 871-881.

Wauthier, E.L., P.C. Hanawalt, and J.M. Vos. 1990. Differential repair and replication of damaged DNA in ribosomal RNA genes in different CHO cell lines. J. Cell. Biochem. 43: 173183.

Weeda, G., R.C.A. van Ham, W. Vermeulen, D. Bootsma, A.J. van der Eb, and J.H.J. Hoeijmakers, 1990. A presumed DNA helicase encoded by ERCC-3 is involved in the human repair disorders xeroderma pigmentosum and Cockayne's syn- drome. Cell 62: 777-791.

Weeks, G., S.E. Hardin, J. Shen, J.M. Lee, and A.L. Greenleaf. 1993. Locus-specific variation in phosphorylation state of RNA polymerase II in vivo: correlations with gene activity and transcript processing. Genes \& Dev. 7: 2329-2344.

Yokomori, K., A. Admon, J.A. Goodrich, J.-L. Chen, and R. Tiian. 1993a. Drosophila TFIIA-L Is processed into two subunits that are associated with the TBP/TAF complex. Genes \& Dev. 7: 2235-2245.

Yokomori, K., J.-L. Chen, A. Admon, S. Zhou, and R. Tjian. 1993b. Molecular cloning and characterization of $\mathrm{dTAF}_{\mathrm{II}} 30 \alpha$ and $\beta$ : Two small subunits of Drosophila TFIID. Genes \& Dev. 7: 2587-2597.

Zawel, L. and D. Reinberg. 1993. Initiation of transcription by RNA polymerase II: A multistep process. Prog. Nucleic Acid Res. Mol. Biol. 44: 67-108.

Zhou, Q., P.M. Lieberman, T.G. Boyer, and A.J. Berk. 1992. Holo-TFIID supports transcriptional stimulation by diverse activators and from a TATA-less promoter. Genes \& Dev. 6: 1964-1974. 


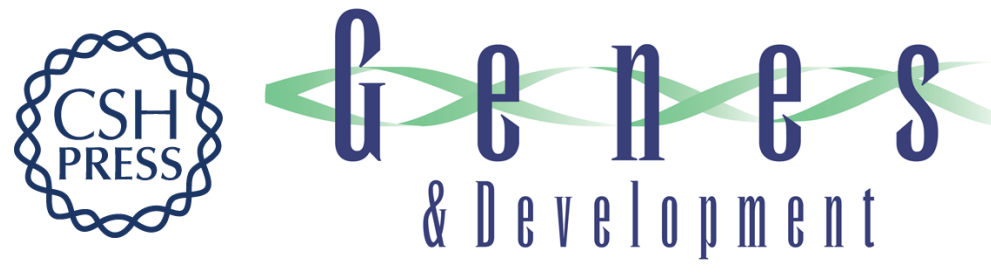

\section{Transcription factor IIE binds preferentially to RNA polymerase Ila and recruits TFIIH: a model for promoter clearance.}

M E Maxon, J A Goodrich and R Tjian

Genes Dev. 1994, 8:

Access the most recent version at doi:10.1101/gad.8.5.515

References This article cites 43 articles, 23 of which can be accessed free at:

http://genesdev.cshlp.org/content/8/5/515.full.html\#ref-list-1

License

Email Alerting

Service

Receive free email alerts when new articles cite this article - sign up in the box at the top right corner of the article or click here.

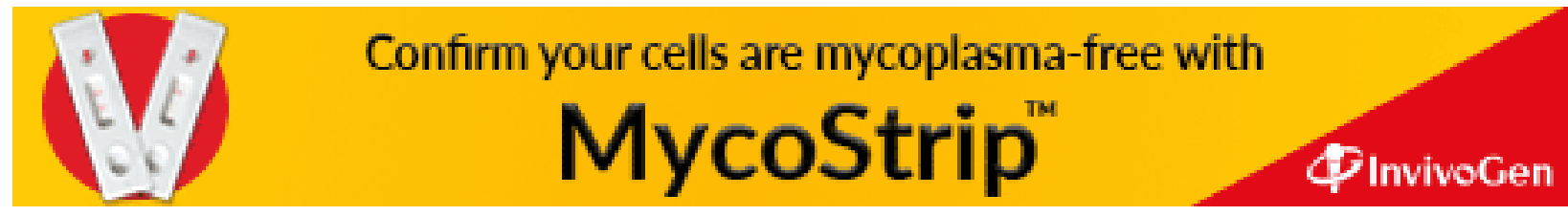

